\title{
Deformation and three-dimensional displacement of fibers in isometrically contracting rat plantaris muscles.
}

Citation for published version (APA):

Savelberg, H. H. C. M., Willems, P. J. B., Baan, G. C., \& Huijing, P. A. (2001). Deformation and threedimensional displacement of fibers in isometrically contracting rat plantaris muscles. Journal of Morphology, 250(1), 89-99. https://doi.org/10.1002/jmor.1061

Document status and date:

Published: 01/01/2001

DOI:

10.1002/jmor.1061

Document Version:

Publisher's PDF, also known as Version of record

Document license:

Taverne

Please check the document version of this publication:

- A submitted manuscript is the version of the article upon submission and before peer-review. There can be important differences between the submitted version and the official published version of record.

People interested in the research are advised to contact the author for the final version of the publication, or visit the DOI to the publisher's website.

- The final author version and the galley proof are versions of the publication after peer review.

- The final published version features the final layout of the paper including the volume, issue and page numbers.

Link to publication

\footnotetext{
General rights rights.

- You may freely distribute the URL identifying the publication in the public portal. please follow below link for the End User Agreement:

www.umlib.nl/taverne-license

Take down policy

If you believe that this document breaches copyright please contact us at:

repository@maastrichtuniversity.nl

providing details and we will investigate your claim.
}

Copyright and moral rights for the publications made accessible in the public portal are retained by the authors and/or other copyright owners and it is a condition of accessing publications that users recognise and abide by the legal requirements associated with these

- Users may download and print one copy of any publication from the public portal for the purpose of private study or research.

- You may not further distribute the material or use it for any profit-making activity or commercial gain

If the publication is distributed under the terms of Article $25 \mathrm{fa}$ of the Dutch Copyright Act, indicated by the "Taverne" license above, 


\title{
Deformation and Three-Dimensional Displacement of Fibers in Isometrically Contracting Rat Plantaris Muscles
}

\author{
Hans H.C.M. Savelberg, ${ }^{1 *}$ Paul J.B. Willems, ${ }^{1}$ Guus C. Baan, ${ }^{2}$ and Peter A. Huijing ${ }^{2,3}$ \\ ${ }^{1}$ Department of Human Movement Sciences, Faculty of Health Science, Maastricht University, \\ Maastricht, The Netherlands \\ ${ }^{2}$ Instituut voor Fundamentele \& Klinische Bewegingswetenschappen, Faculteit Beweginswetenschappen, \\ Vrije Universiteit, Amsterdam, The Netherlands \\ ${ }^{3}$ Integrated Biomedical Engineering for Restoration of Human Function, Department of Biomedical \\ Mechanical Engineering, Universiteit Twente, Enschede, The Netherlands
}

\begin{abstract}
In this study, the deformation of different fibers of the rat $m$. plantaris during "isometric" contractions at different muscle lengths was considered. Because the $m$. plantaris has an obviously inhomogeneous architecture, its fibers on the medial side of the muscle belly are judged to be shorter than those on the lateral side of it. It was expected that longitudinal deformation of different fibers would vary accordingly. A 3D video analysis of contracting muscle showed that longitudinal strain of fibers as a function of muscle length does not differ between fibers on different sides of the muscle. Apart from longitudinal shortening, the fibers were also displaced laterally during a contraction. The fibers displaced during a con-
\end{abstract}

It often has been proven and is widely accepted that muscle geometry is of considerable importance to the function of a muscle. The degree of pennation, the ratio between fiber length and muscle length, is used as a measure for muscle geometry (Woittiez et al., 1983). Huijing (1996) stated that muscle geometry is one of the aspects of muscle architecture, the arrangement of the elements constituting the muscle. In this context (for review see Huijing, 1996), distribution of fiber mean sarcomere length was shown to be important. It affects the length range in which a muscle can produce force (Willems and Huijing, 1994). Therefore, a muscle is not to be considered a collection of numerous identical fibers.

Fibers can be different, not only with respect to microscopic characteristics, but also their orientation and length may vary in a muscle (Benninghoff and Rollhäuser, 1952; Sacks and Roy, 1982; Callister and Peterson, 1992; Eijden and Raadsheer, 1992; Willems and Huijing, 1994). Based on this, Savelberg and Schamhardt (1995) designed a model to predict the effect of macroscopic inhomogeneity within a muscle on its force-length characteristics. The model showed that differences in fiber lengths in different sagittal planes of a muscle affect the traction in a direction perpendicular to their longitudinal axis. The displacement of the fibers occurred asymmetrically, resulting in a helical deformation of the whole muscle. It is concluded that the asymmetric displacement and the helical deformation must result from transversal forces between the fibers. It is hypothesized that these transversal forces cancel out differences in longitudinal strains that might exist between fibers. J. Morphol. 250: 89-99, 2001. @ 2001 Wiley-Liss, Inc.

KEY WORDS: muscle architecture; muscle fiber shortening and displacement; transversal force

force-length relationship, and thus the function of a muscle. The model by Savelberg and Schamhardt (1995) simply summed up the forces generated by different muscle parts having a different geometry, and therefore the model lacked any interaction (transverse or otherwise) between muscle parts. In a macroscopically inhomogeneous and asymmetric muscle, transverse deformations may be introduced either due to different force-generating capacities in different parts of the muscle, or due to forces being transmitted along parallel pathways in the muscle (Huijing et al., 1998; Huijing, 1999). These transverse deformations and forces have functional relevance, not only because they affect the force production of the muscle itself, but also because they affect the characteristics of adjacent muscles by deflecting their line of pull. If muscles share a common tendon, this kind of interaction is even more prominent. Van Donkelaar et al. (1999) showed that the presence of transverse effects is not limited to muscle with an

\footnotetext{
*Correspondence to: H.H.C.M. Savelberg, PhD, Department of Human Movement Science, Faculty of Health Sciences, Maastricht University, P.O. Box 616, NL-6200 MD Maastricht, The Netherlands. E-mail: hans.savelberg@bw.unimaas.nl
} 
inhomogeneous morphology, noting that even in the relatively homogeneous rat $m$. gastrocnemius, asymmetries in transverse strains exist.

To study effects of macroscopic inhomogeneity on the contraction and displacement of a whole muscle and of individual fibers within the muscle, threedimensional (3D) deformation of the rat $m$. plantaris was studied for several fibers. M. plantaris is clearly macroscopically inhomogeneous (Fig. 1). Its aponeuroses are not parallel, but are at an angle with respect to each other in the plane perpendicular to the line of action of the muscle. This results in fibers on one side of the muscle being longer and having lower fiber angles than fibers on the other side. In this way, the $m$. plantaris seems to be a natural representation of the kind of inhomogeneity that was simulated in the model by Savelberg and Schamhardt (1995).

It was hypothesized that due to macroscopic inhomogeneity of this muscle, fibers at different sites of the muscle would undergo different strains during contractions, and that the strains of different fibers would differ as a function of muscle length. Given different deformations at different sites in the muscle, it was hypothesized that fibers would not only deform longitudinally, but in addition would be subjected to lateral displacements.

\section{MATERIALS AND METHODS}

Six female Wistar-Kyoto rats (body mass 153-201 g; age 10-11 weeks; mass of $m$. plantaris $0.29-0.36$ g) were anesthetized before the experiments by an intraperitoneal injection of sodium pentobarbital solution (initial dose $54 \mathrm{mg} \cdot \mathrm{kg}^{-1}$ ). During the experiments, additional anesthetic injections (dose 10 $\mathrm{mg} \cdot \mathrm{kg}^{-1}$ ) were provided if necessary. The experiments were in full agreement with Dutch law on animal experimentation and were approved by a university ethics committee.

\section{Surgery}

M. plantaris of the animals was exposed by removing the skin, $m$. biceps femoris, $m$. soleus, and the medial head of the $m$. gastrocnemius. The calcaneus was cut, maintaining the connection of the bone fragment to the Achilles tendon. The $m$. plantaris shares its proximal aponeurosis with the lateral head of the $m$. gastrocnemius. The fibers of the lateral head of the $m$. gastrocnemius were carefully cut from this aponeurosis. The innervation and blood flow to the $m$. plantaris and the origin of the muscle at the femur were preserved. Close to the sacral plexus, the $n$. ischiadicus, which innervates the $m$. plantaris, was cut from the rest of the central nervous system. Branches of the $n$. ischiadicus to other muscles were cut as well. During the operation and the experiment, the animal was positioned on a heated surface $\left(37.0 \pm 0.1^{\circ} \mathrm{C}\right)$. Every $5 \mathrm{~min}$, Ringer's solution was applied to the muscle by a syringe to moisten it.

\section{Experimental Procedure}

The ends of six fibers on the surface of the muscle were marked with fluorescent polystyrene spheres ( $\varnothing 0.5-0.7 \mathrm{~mm}$; Bangs Laboratories Inc., Fishers, USA). The spheres were attached by tissue glue (Histoacryl blau ${ }^{\circledR}$, Braun, Melsungen, Germany). In addition, each fiber was marked by at least two more spheres at evenly distributed distances between the markers on the fiber ends. Although it is known that the presence of an intact epimysium may interfere with assessment of fiber deformation (Scott and Loeb, 1995), the epimysium over the $m$. plantaris, which is very thin, was not removed. It was assessed whether the markers moved with the fiber and not with the epimysium. Two fibers were on the medial aspect of the muscle, two on the lateral side, and two on the distal surface (Fig. 1). The proximal aponeurosis is more or less rectangular shaped. The marked fibers on the distal surface originate from the distal corners of the proximal aponeurosis. Both fibers insert at the most distal point of the muscle belly. The marked fibers at the medial and lateral aspect originate from the medial and lateral border of the proximal aponeurosis, respectively. The two fibers selected on each side of the muscle divided the medial and lateral aspect of the muscle into three equal parts.

Two clamps were attached to the femur to connect it to the table of a tensile-testing machine (Zwick 1445, Zwick GmbH, Ulm, Germany). The calcaneus was connected to a force transducer, the displacement of which could be controlled and measured (accuracy $0.01 \mathrm{~mm}$ ). The orientation of the m. plantaris relative to the femur was similar to an in vivo situation in which the knee angle is $90^{\circ}$. A bipolar stimulation electrode was attached to the $n$. ischiadicus, through which the nerve could be stimulated $(100 \mathrm{~Hz}$, with $1.3 \mathrm{~V}$ pulse trains consisting of $0.6 \mathrm{~ms}$ duration square pulses) using a pulse generator (HSE 215/IZ, Freiburg, Germany). At different lengths, the muscle was activated for $300 \mathrm{~ms}$. During a contraction the muscle was held at constant length, while length changes of tendon and muscle fibers were possible. Between contractions the muscle was allowed to rest for at least $3 \mathrm{~min}$. The muscle length at subsequent contractions was randomly determined.

\section{Data Acquisition}

In order to measure muscle and fiber deformation, the $3 \mathrm{D}$ positions of the spheres were recorded at a sampling rate of $50 \mathrm{~Hz}$ by two synchronized CCD cameras (MX5, Adimec, The Netherlands) equipped with $75 \mathrm{~mm}$ lenses (Ernitec F1.3 TV) and with a yellow filter $(600 \mathrm{~nm})$. The images were digitized 


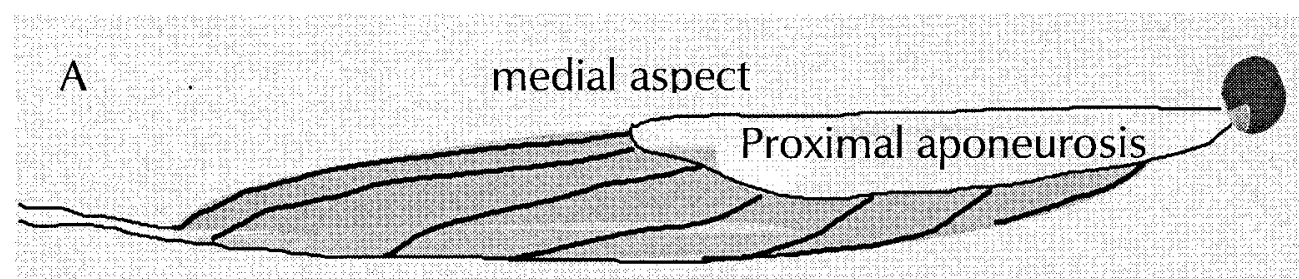

distal

lateral aspect

proximal

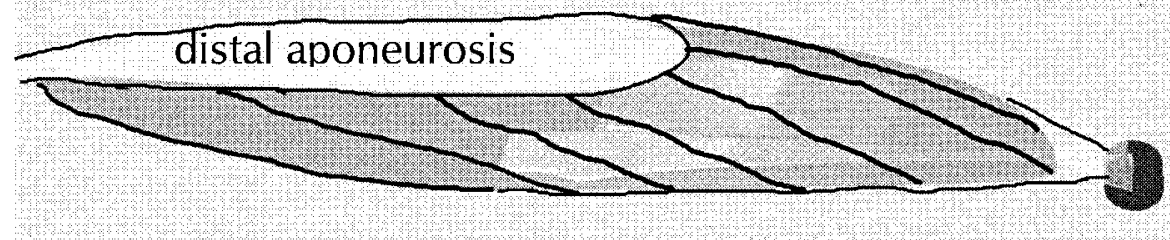

dorsal

$\stackrel{\leftrightarrow}{\longrightarrow}$

medial

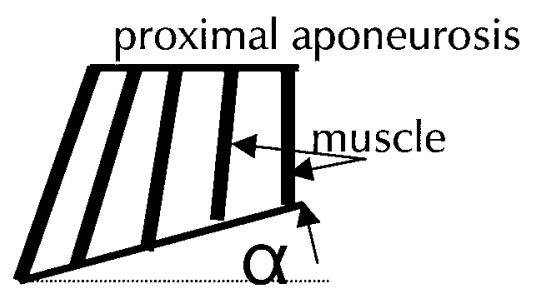

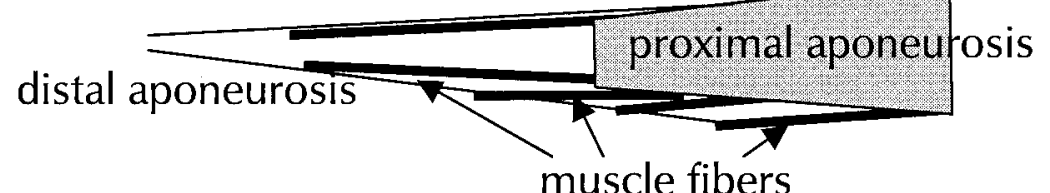

medial

muscle fibers

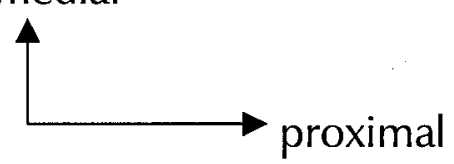

C: dorsoventral view

distal aponeurosis

\section{B: proximodistal view}

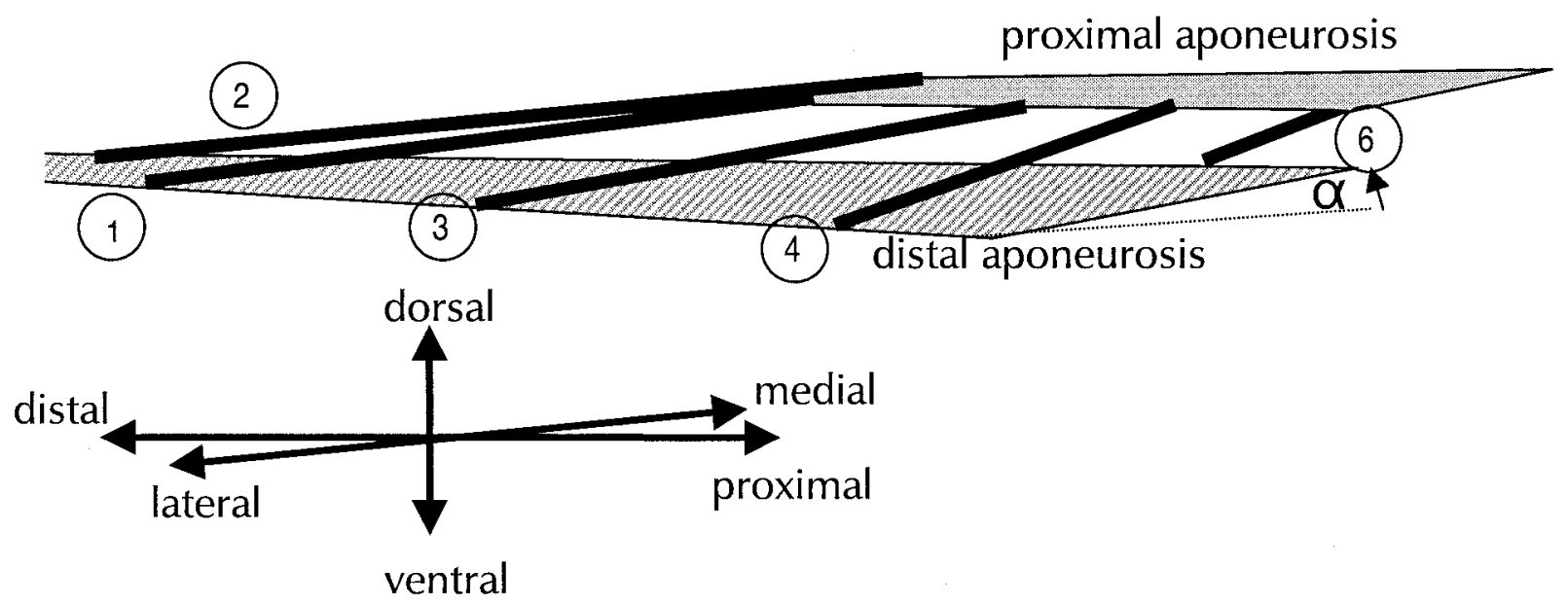

D: 3D schematic impression

Fig. 1. Schematic illustrations of the geometry of rat $m$. plantaris. In (B) the projection on the transversal plane is shown (proximodistal view), illustrating the angulation of the proximal and distal aponeurosis relative to each other. (C) gives the projection on the frontal plane. In (D) the location of marked fibers is indicated: Five of the six fibers considered have been drawn (black lines). The numbers in the circles correspond to the numbers of fibers used in Table 1 and Figure 6. Fiber 5 is located on the medial side between fiber 2 and fiber 6 . The hatched area represents the inside of the distal aponeurosis; the grey area is the outside of the proximal aponeuroses. Relative to the proximal aponeuroses, the distal aponeurosis is angulated $(\alpha)$ to the frontal plane. 


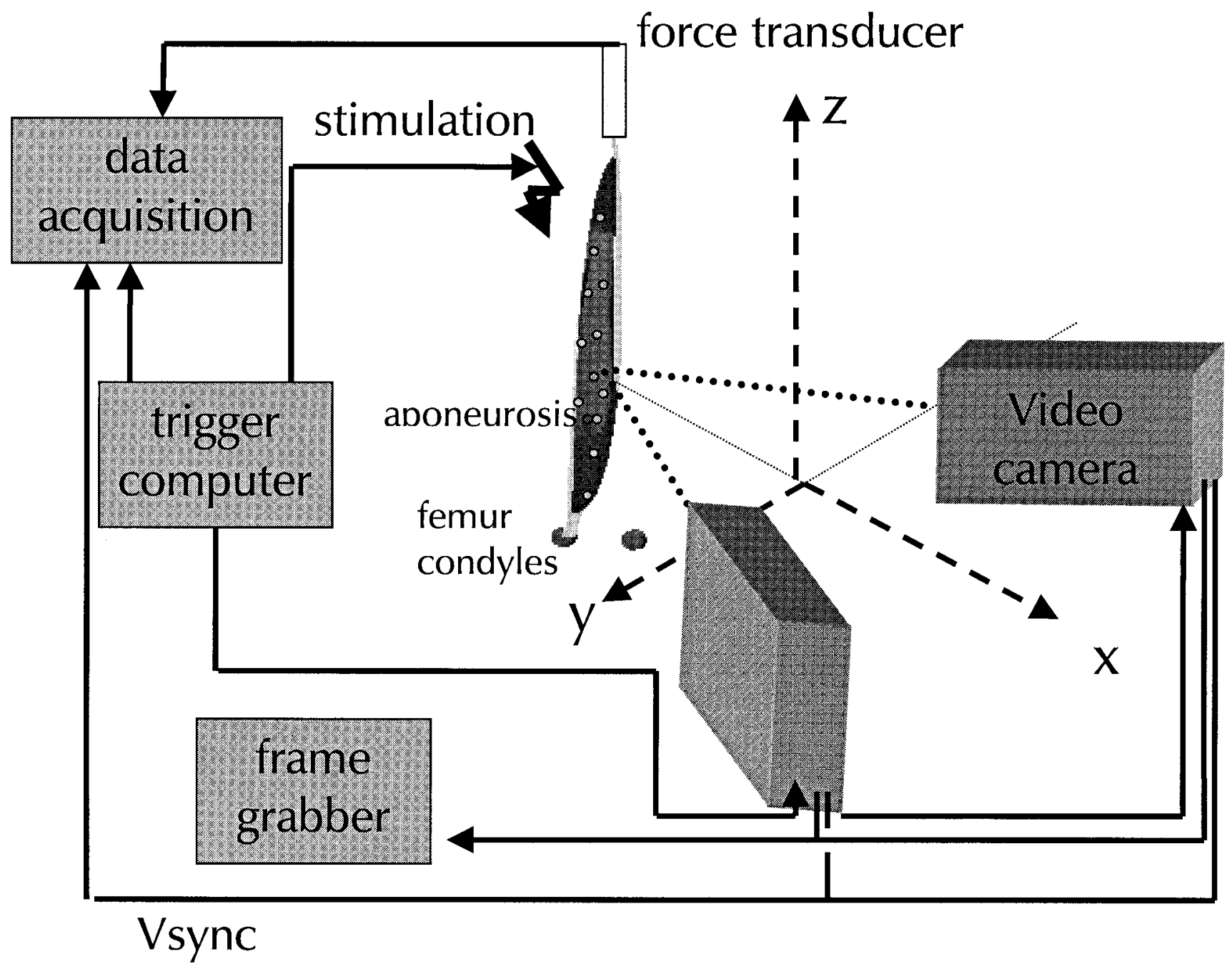

Fig. 2. Schematic representation of the experimental design. The position of both video cameras with respect to the muscle and the orientation of the coordinate system is shown. The z-axis represents the longitudinal direction of the muscle. The representations of the $\mathrm{x}$-axis and the $\mathrm{y}$-axis change as the animal and the muscle are rotated with respect to the cameras in order to view other parts of the muscle. Furthermore, the connections between the trigger computer, frame grabber, and data-acquisition computer are shown.

real-time and stored on a computer via the Red and Green input of an 8-bit RGB frame grabber $(400 \times$ 512 pixels; DMA Magic, Matrox, UK). To optimize the marker detection, the muscle was illuminated by a light source (KL 1500 electronic, Schott, Wiesbaden, Germany) equipped with a blue filter $(450 \mathrm{~nm})$. The angle between the cameras amounted to approximately $60^{\circ}$. The distance to the object was about $0.9 \mathrm{~m}$. The line through the centers of the lenses was defined as the $\mathrm{x}$-axis of the coordinate system; the perpendicular y-axis crossed the $\mathrm{x}$-axis at the intersection point of the optical axes of the cameras. The vertical z-axis was perpendicular to both the $\mathrm{x}$ - and $\mathrm{y}$-axis, and was parallel to the longitudinal direction of the muscle (Fig. 2). Due to the curvature of the muscle it was not possible to view all markers with both cameras from one side. Therefore, to be able to measure marker displacement at all sides of the muscle, the rats were rotated to obtain three different muscle positions relative to the cameras. In this way the muscle was viewed from its medial, dorsal, and lateral aspect. At each of these three positions the muscle was held at a range of different lengths while stimulated. The order in which the different viewpoints were considered was random.

Force was measured by a force transducer (accuracy $0.02 \mathrm{~N}$; Zwick $\mathrm{GmbH}$ ) and $\mathrm{AD}$-converted at $1000 \mathrm{~Hz}$ (8-channel, 12-bits Lab-NB AD-conversion board, National Instruments). To link the deformation measurements and the force recordings, the Vsync (video synchronization) signal of the cameras was sampled simultaneously.

A computer was used to trigger simultaneously the CCD cameras and the data-acquisition computer, and with a $150 \mathrm{~ms}$ delay, the stimulation of the nerve. 


\section{Data Processing}

Using image processing software (Timwin 1.3, TEA, Dordrecht, The Netherlands), geometrical marker centroids were assessed in the digitized images. From these centroids, 3D positions of each of the markers were reconstructed (Muijtens et al., 1995). The protocol that was used calculates the positions of the cameras based on stereoscopic pairs of displacement tracks during a contraction of all the markers. This protocol did not need an a priori calibrated camera setup. Due to the curvature of the muscle it was not always possible to see all markers attached to one fiber in both cameras.

Manually, the markers were selected that were marking one and the same fiber. The distances between subsequent markers on a fiber were calculated for each video frame. Thus, for each fiber the length was calculated at different instants during a contraction at a particular length of the muscle. The procedure was repeated at several muscle lengths. Fiber length was estimated in two ways. Summation of all distances between subsequent markers on a fiber yields estimates of the actual length of that fiber. Calculation of the distance between the most proximal and the most distal marker of a fiber yields an estimate of the linearized fiber length. By comparing the differences between these two estimates of fiber length, the effect of curvature of a fiber on its length estimates could be obtained. Furthermore, the presence of several markers on a fiber allowed comparison of values for proximal, middle, and distal segments of a fiber.

The deformations of muscle and of the marked fibers were investigated by:

1. Assessing the length change, as well as displacement of a fiber, during the dynamics of force build up. To assess the effect of muscle length on the development of force, force-time traces were compared at muscle optimum length and at the short and long lengths at which the muscle developed $50 \%$ of its optimum force. For this comparison, to remove any effect of different absolute force values, for each length active force-time traces were normalized for peak active force at that length.

2. Determining the deformation and displacement of a fiber during the tetanus "plateau" for different lengths of the muscle. For each muscle optimum length was assessed, which was the length at which the muscle developed its maximal force. Optimum muscle length was determined by fitting a polynomial for data relating active peak forces for each tetanus plateau to muscle length. Active peak force was calculated by subtracting passive force, which was measured before onset of stimulation. Muscle length was defined as the length of the muscle-tendon complex, and was measured as the displacement of the force transducer. Regression analysis was applied to assess the degree of the polynomial. The degree ranged between three and five. The optimum muscle length was defined as the length at which this polynomial reached its maximum. Subsequently, for each fiber a polynomial was fitted for the length of the fiber as a function of the length of the muscle. Typically, the degrees of these polynomials varied between one and three. Evaluating this polynomial for the optimum muscle length yielded the reference length of a fiber.

Strain of a fiber as a function of muscle length was calculated by dividing the change of its actual length from its reference length by the reference length of the fiber.

\section{Statistics}

ANOVA for repeated measurements, being the different lengths (one-way with sample number as a factor), was performed to test effects of muscle length on the shape of normalized force-time traces. If the interaction between different lengths and sample numbers is significant, it can be concluded that the shapes of the curves at different lengths differ significantly. Similarly, fiber strain at the plateau of the tetanus was evaluated for marked fibers.

ANOVAs for repeated measurements were used also to compare fiber length and displacement of fibers at three muscle lengths: short length (at which active peak force is approximately 50\% of optimal force), optimum muscle length, and long length (active peak force of contraction is approximately $50 \%$ of optimal force).

A paired $t$ test was used to test effects of taking fiber curvature into account on the fiber length (change) assessed. To evaluate differences in strain between proximal, middle, and distal parts of a fiber, a one-way ANOVA procedure was followed.

For each test the level of significance was chosen as $P<0.05$.

\section{RESULTS \\ Active Force Development During a Contraction}

Force profiles during contractions show significant effects $(P<0.001)$ of muscle length (Fig. 3$)$. At lengths below optimum length, force increases steeply in time following onset of stimulation, and after having reached its peak value, it decreases rather quickly as well. At lengths near optimum length, the initial increase is similarly steep, but the peak value is held until the stimulation is switched off. At lengths over optimum length, the rate of force development following stimulation of the muscle is considerably decreased compared to shorter lengths.

\section{Effects of Fiber Curvature}

All marked fibers were provided with at least four markers. However, not all markers were visible dur- 

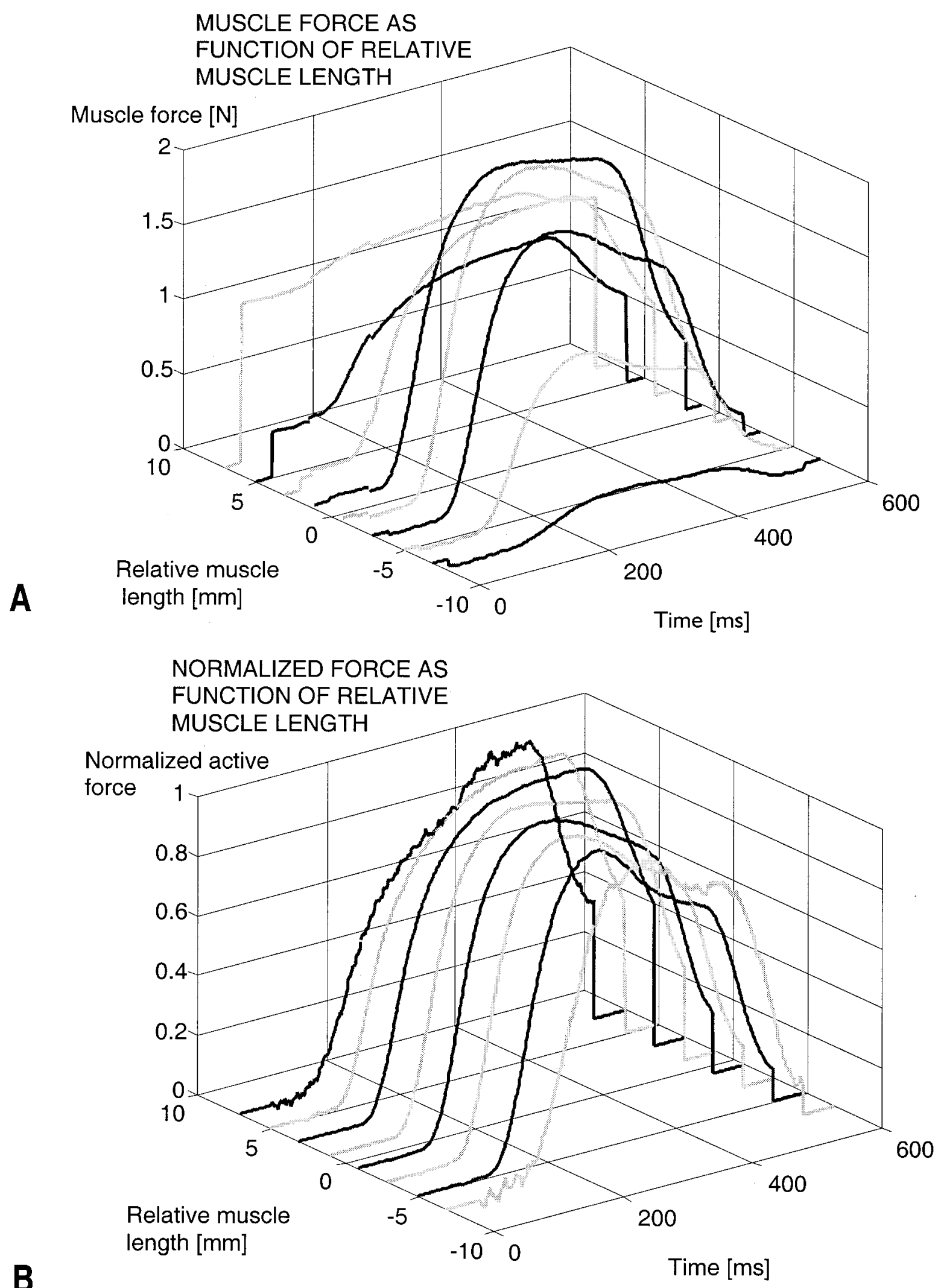

Fig. 3. The effect of muscle length on force-time traces. Muscle length is presented as deviation from optimum muscle length (i.e., muscle length of maximum muscle force). Force was sampled at $1000 \mathrm{~Hz}$, stimulation started at $\mathrm{t}=150 \mathrm{~ms}$. The different gray shading in subsequent curves only serves to increase legibility of the figure. A: Effects on absolute force time traces. B: Effect on normalized force-time profiles. Muscle force is normalized for its peak value at each muscle length. This normalization allows comparison of the shape of the profiles as a function of muscle length. 
TABLE 1. Number of markers analyzed for each fiber in each of the muscles

Fiber 1 Fiber 2 Fiber 3 Fiber 4 Fiber 5 Fiber 6

\begin{tabular}{lllllll}
\hline Plantaris 02 & 4 & 2 & 2 & 4 & - & 2 \\
Plantaris 03 & 8 & 8 & 3 & 5 & 4 & 3 \\
Plantaris 04 & 8 & 4 & 2 & - & 3 & - \\
Plantaris 05 & 7 & 3 & 5 & 3 & - & - \\
Plantaris 06 & 5 & 6 & - & 3 & 3 & 4 \\
Plantaris 07 & 4 & 4 & 4 & 4 & 2 & 3
\end{tabular}

ing a complete contraction at all muscle lengths. Due to the curvature of the muscles, some markers could not be seen simultaneously by both cameras, or the markers moved out of view during a contraction. In Table 1, for each fiber for each animal the number of markers are listed that were used for analysis.

Analysis of marked fibers at three muscle lengths reveals that accounting for the curvature of the fiber yields significantly longer muscle fibers $(P<0.01)$. At optimum muscle length, the mean increase is $1.8 \%$. At short and long lengths, with active force at $50 \%$ of optimal force, fiber lengths are $2.5 \%$ and $0.7 \%$ larger, respectively, if curvature is accounted for. Accordingly, strain at these short and long muscle lengths is significantly less $(P<0.01)$, by $0.9 \%$ and $0.6 \%$, respectively, if taking curvature into account.

No significant effect of fiber segment (proximal, middle, or distal) on strain can be shown.

Based on these results, we decided to consider (see below) only the lengths and strains between the most proximal and the most distal marker, i.e., to neglect effects of curvature.

\section{Shortening of Fibers Following Stimulation}

During a contraction, no systematic (significant) differences in mean longitudinal strain can be shown between fibers studied at different locations within the muscle.

However, mean longitudinal fiber strain depends significantly $(P<0.01)$ on muscle length (Fig. 4$)$. At a short length, with force equaling $50 \%$ of optimal force, fibers shorten by $9.2 \pm 6.1 \%$ during the first $150 \mathrm{~ms}$ following stimulation. Subsequently, the fibers remain at these lengths until the stimulation is switched off. At muscle optimum length, fibers shorten during the contraction by $4.7 \pm 3.8 \%$. At a long length of the muscle, yielding $50 \%$ of optimal force, on activation, fiber length decreases during the contraction by $0.1 \pm 0.4 \%$. Shortening of muscle fibers on activation demonstrates that the isometric contractions on the level of the muscle are not at all muscle lengths represented by isometric contractions at the level of the fiber. Especially at the shorter lengths, the changes in muscle fiber length are considerable, and the fibers undergo concentric contractions before becoming isometric. At the longer lengths (i.e., longer than optimal), muscle fiber length does not change, implying an isometric contraction at the level of the muscle fiber.

In addition to the conventional assessment of fiber length changes, the $3 \mathrm{D}$ analysis of marker positions allows the registration of fiber displacement during a contraction. Following onset of stimulation, the fibers are displaced in the plane perpendicular to the longitudinal direction of the muscle (Fig. 5A-C shows a typical example). This displacement is larger than the length change of the muscle fiber. Also, a stimulation is sustained and fibers do not shorten any further, a displacement of the fiber in the xy-plane can still be noticed (Fig. 5D-F). The lateral displacement immediately following stimulation, as well as the displacement during prolonged stimulation, is largest at short muscle lengths and decreases if longer initial lengths are imposed on the muscle. The dorsal fibers move medially and ventrally, the lateral fibers shift dorsally, and the medial fibers take on a more ventral position. The lateral displacement of fibers during contractions illustrates that forces perpendicular to the longitudinal direction of the fibers are present in the muscle. These forces may originate from variations in the orientation of fibers over the muscle or from forces transmitted by lateral connections between fibers and the intramuscular connective tissue.

It is striking that the lateral displacement shows a general pattern similar to that of the force development (e.g., Fig. 3): rising steeply and decreasing before the stimulation is ended at short muscle lengths, and rising and decreasing moderately at long muscle lengths. The longitudinal strain of the fibers, their shortening, does not follow this specific

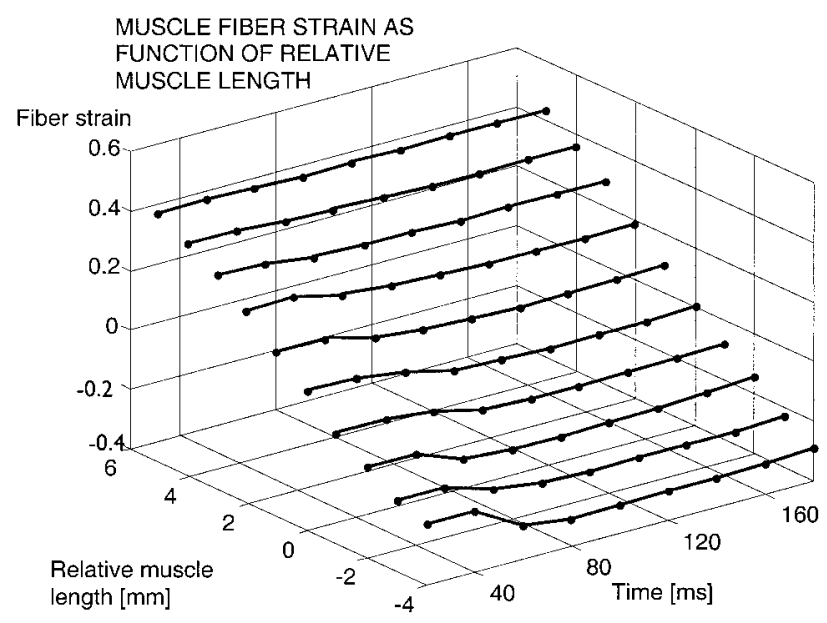

Fig. 4. Fiber strain during a contraction as a function of muscle length. Strain is defined as the change of the fiber length with respect to the reference length divided by the reference length (i.e., fiber length at optimum muscle length when the plateau in the force-time profile was reached). The sample frequency of the video was $50 \mathrm{~Hz}$. Only the fiber strain at the sampling instant immediately preceding onset of stimulation and at the eight samples following onset of stimulation are presented. 
FIBER DEFORMATION AND DISPLACEMENT

DURING CONTRACTIONS
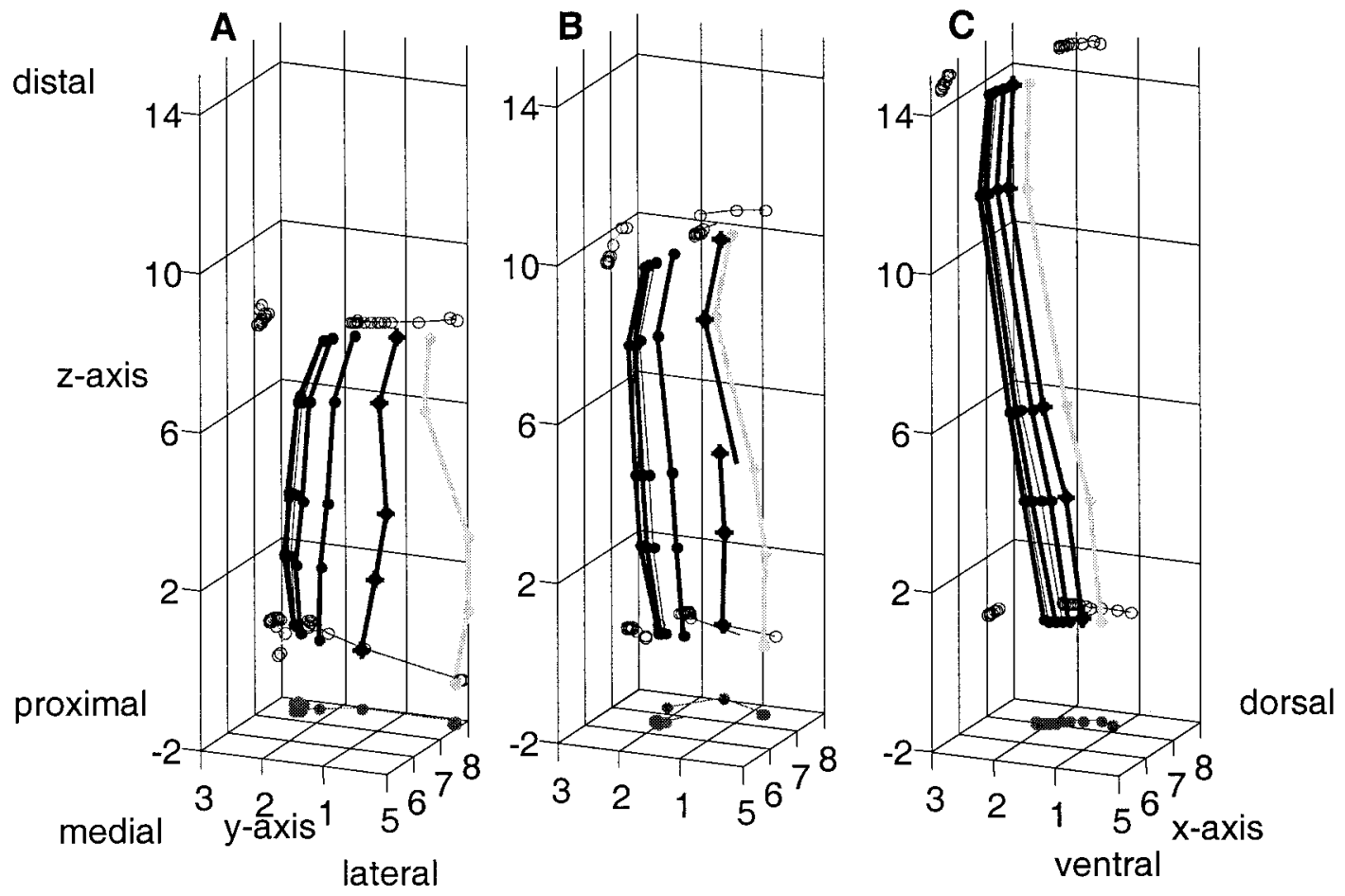

RELATIVE SHORTENING AND DISPLACEMENT

DURING A CONTRACTION FOR THREE MUSCLE LENGTHS

D

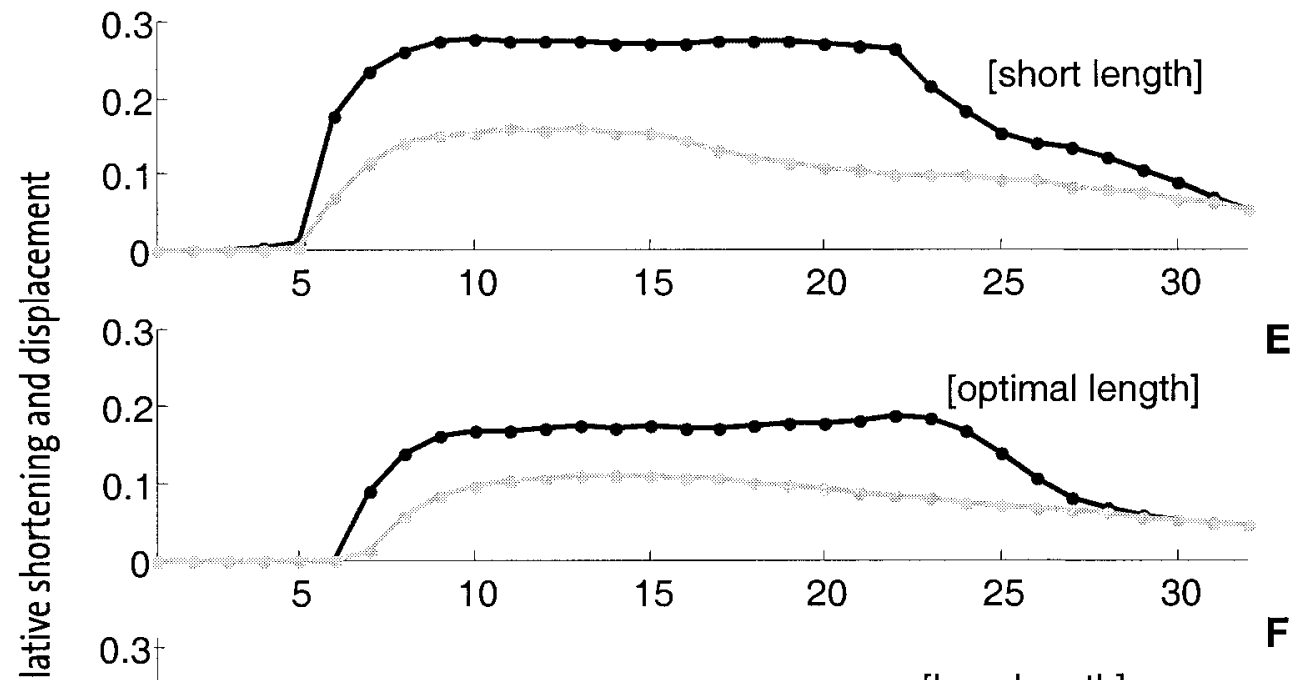

[long length]

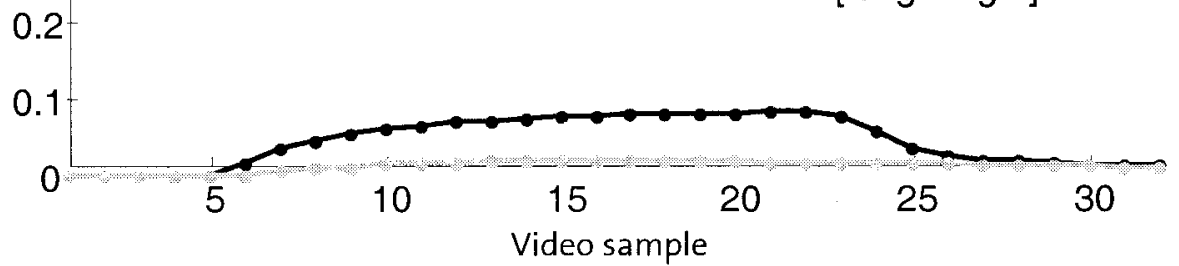

Figure 5. 
pattern. Neither does it reverse immediately as the stimulation is switched off. The similarity between muscle force and lateral displacement of fibers was not statistically evaluated. However, it suggests that fiber displacement and muscle force are related more closely than muscle force and shortening of a single fiber.

\section{Effects of Muscle Length on Isometric Fiber Length}

Fibers at different locations within the muscle show similar mean longitudinal strains at the instant of peak force during the tetanus plateau. However, strain is a function of muscle length (Fig. 6 shows a typical example). At the short muscle length, at which active force approaches zero, maximal fiber strain attains $-40 \%$. Maximal fiber strain found at the longest muscle length studied amounts to a value of up to $60 \%$.

These mean longitudinal strain patterns differ somewhat between muscles, but are fairly constant between different fibers within one muscle.

\section{DISCUSSION}

In this study two intriguing phenomena were found: fibers were found to move out of their line of action, and the strain of muscle fibers was found to be independent of their location on the muscle surface. The study also showed that force profiles depend upon muscle length.

Fig. 5. A typical example of deformation and displacement of a fiber during contraction at three different muscle lengths. A-C: (A) short length, (B) the near optimum length, and (C) long length. Optimum muscle length was defined as the length of maximal active force. In this case, "near optimum length" represents a length corresponding to approximately $95 \%$ of maximal active force, and "short length" represents a length corresponding to $50 \%$ of maximal active force. At "long length," the muscle was stretched to a length at which $70 \%$ of maximal active force was exerted.

In each panel, the z-axis coincides with the line of action of the muscle, which, as it turns out, approximates the longitudinal orientation of the fiber considered. In this figure, the y-axis approximates the transversal axis (medial-lateral); the x-axis approximates the sagittal axis (ventral-dorsal). The different lines in each panel connect five markers on one fiber. Each of the lines shows the markers on the fiber at subsequent instants within a contraction (20 ms apart in time). The gray line represents the fiber position at the last video-image preceding stimulation (passive fiber). The black line with the crossed markers shows the fiber at the first image following stimulation. The dots and circles represent the projection of the most distal and the most proximal markers, respectively, on the yz-, yx-, and zx-planes. D-F: The relative shortening (in gray) and displacement (in black) in the plane perpendicular to the longitudinal direction of the muscle during a contraction at three different muscle lengths: short length (D), near optimum length $(\mathbf{E})$, and long length $(\mathbf{F})$. The deformation (shortening is positive) and displacement are presented as a fraction of the fiber length before onset of stimulation.

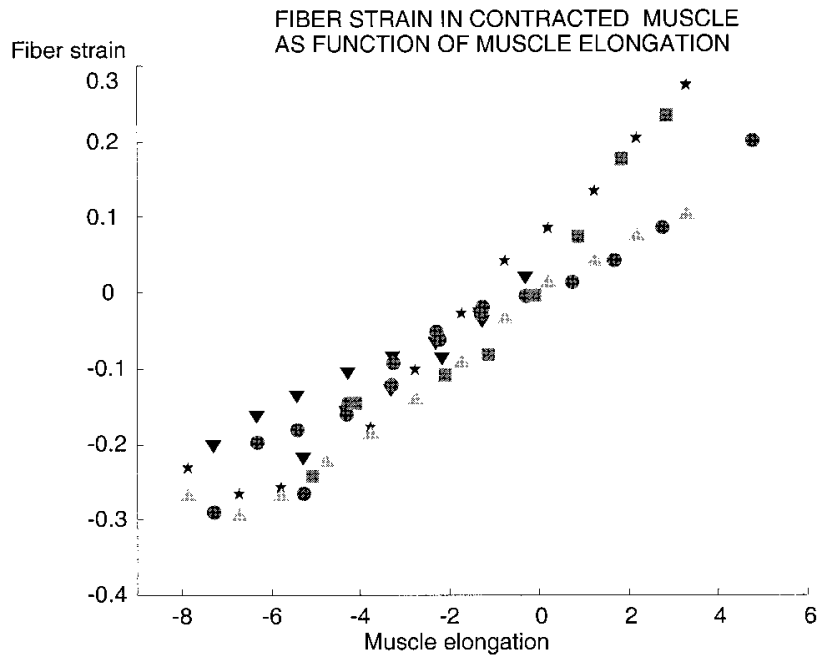

Fig. 6. A typical example of fiber strain for different fibers in a muscle, as a function of muscle length. Strain was defined as the length change at the plateau of muscle force, with respect to the optimum length divided by the length of that fiber at the force plateau at optimum length. Different markers represent fibers at different locations within the muscle. The positions of the fibers considered are illustrated in Figure 1. The filled circles represent the lateral fiber on the dorsal aspect of the muscle (fiber 1), the dark downward pointing triangles represent the medial fiber on the dorsal aspect (fiber 2), the pentagrams represent the distal fiber on the lateral aspect of the muscle (fiber 3), the grey-shaded, upward pointing triangles represent the proximal fiber on the lateral aspect (fiber 4), and the squares represent the distal fiber on the medial aspect of the muscle (fiber 5). For this muscle, no strain data for the proximal fiber on the medial aspect of the muscle (fiber 6) were available.

\section{Force Profiles}

The profiles of force-time curves of contracting muscles were found to depend upon initial muscle length in two aspects: the rate of force development and the ability to maintain force at a tetanic plateau. The early decrease of force production in muscle at short length can be explained by the fact that the contraction is concentric at short muscle lengths (Fig. 4). At shorter lengths the contractile velocity is high, which results in a rapid decline of the force. With increasing muscle lengths velocity is reduced to zero and so is the force decline. Furthermore, the concentric contraction of fibers shorter than optimum length will result in even shorter fibers and thus in less force generation. Comparable results, concentric shortening sarcomeres during isometric contractions due to compliance of the series elasticity of a muscle, have also been reported by Griffiths (1991) and Van Donkelaar et al. (1999).

A difference in rate of force development with differing muscle lengths is also seen in the force profiles. At long lengths when the system should be stiffest the force develops slowly; at short lengths a rapid development is seen. Obviously, the sarcomere length dependence on the excitation dynamics of the muscle fibers (Balnave and Allen, 1996) must be responsible for this effect. 
The dependence of force profiles upon initial muscle length demonstrates that length of muscle affects motor control in two ways. First, because the magnitude of force differs with sarcomere length, the amount of muscle to be activated has to be adjusted. Second, as the force development is affected by muscle length, the timing of muscle activity should be tuned also.

\section{Muscle Fiber Deformation}

The macroscopic inhomogeneity based on which the $m$. plantaris was selected for this study is not reflected in the differences in strains of different fibers as a function of muscle length. Although this might be surprising, it is a fact reported in other studies too. For the m. gastrocnemius of the rat, it was shown in passive (Van Bavel et al., 1996) and active muscle (Van Donkelaar et al., 1999) that longitudinal strains are equal for different sites within the muscle. Zuurbier and Huijing (1993) have demonstrated that the $m$. gastrocnemius has primary distribution of sarcomere length, and thus is inhomogeneous as well. Furthermore, in a finite element study, Van der Linden (1998) considered the strain in different fibers of the $m$. extensor digitorum longus of the rat. In the $2 \mathrm{D}$ plane that was considered in the simulation, this muscle was also morphologically inhomogeneous: proximal fibers were shorter than distal ones. The simulation showed strains in the different fibers to differ minimally.

The fibers considered in the $m$. plantaris in this study had different absolute lengths, so similar strains will have occurred with different absolute length changes in different fibers. As a consequence, fibers will move relative to each other and exert transverse forces. The presence of transverse forces has been shown previously (Street, 1983; Patel and Lieber, 1997; Huijing et al., 1998; Huijing, 1999).

\section{Displacement of the Whole Muscle}

The finding that fibers not only shorten during a contraction but also move out of their original line of action is important in relation to the functional behavior of the muscle. This displacement depends on the location of a fiber. The overall image of muscle movement, resulting from different displacements of different fibers, shows that while contracting the muscle rotates and translates in a helical way, displacing fibers somewhat distally and clearly away from the site where the shared aponeurosis with the lateral head of the $m$. gastrocnemius is located. It is important to note that this helical displacement also occurs at longer muscle lengths, when the fibers do not change their lengths at all. This indicates that this kind of lateral displacement is not solely caused by a slack hanging muscle being stimulated and erecting itself. This helical movement of the isolated muscle suggests that in an intact musculoskeletal system, the actions of different muscles will affect each other mutually through shared aponeuroses, as in the present case, through shear forces transferred by fascia (Huijing et al., 1998), or by forces normal to the muscle surface resulting from muscle displacements. If the lateral $\mathrm{m}$. gastrocnemius had not been removed it would have been pulled aside by the contracting $m$. plantaris, and consequently its length, and thus force-generating capacity, would have been affected, or the $m$. gastrocnemius, when active, could have resisted the movement of the $m$. plantaris, affecting the performance of this muscle.

The lateral displacement of fibers and of the whole muscle can either be caused by variation in the orientation of fibers over the muscle or by forces transmitted by lateral connections between fibers (Street, 1983; Patel and Lieber, 1997; Huijing et al., 1998; Huijing, 1999). In a pennate muscle, fibers are supposed to generate force along their lengths. In this process they change their orientation within the plane of pennation and transmit, also in the plane of pennation, forces to aponeuroses and tendons. The displacements found in the $m$. plantaris are not limited to one plane. Consequently, they result from fibers that not only meet at an angle with the aponeuroses, as in a pennate muscle, but also with other fibers. Thus, an explanation for the observed displacement of fibers could be that the muscle is pennate in two directions. Also, the displacement might be caused by shear forces between fibers. As it has been deduced above, the similarity in strain between different fibers can be seen as an indication for shear forces between fibers in the $m$. plantaris. Street (1983), Huijing et al. (1998), and Huijing (1999) demonstrated the existence of these myofascial forces. Although this study cannot pinpoint what causes the lateral displacement, it shows that it exists. From the lateral displacement found we can conclude that muscle fibers interact with each other, and that the forces that are developed in sarcomeres are not merely transmitted longitudinally through muscle fibers.

The question arises whether the lateral displacement of muscle fibers in the $m$. plantaris results from the obvious inhomogeneity of fiber length, or if it is present in other muscles too. Van Donkelaar et al. (1999) showed that asymmetry in lateral displacement is not limited to obvious inhomogeneous muscles such as $m$. plantaris. They showed that also in the $m$. gastrocnemius, the transverse strains differ from site to site on the muscle surface. However, in that study, displacement of fibers was not considered. No other studies demonstrating lateral displacement of muscle fibers are known. Obviously, this results from the fact that investigations of muscle mechanics usually merely focus on the physiologically more relevant fiber shortening. Future studies have to answer the question of whether lateral displacement occurs in more muscles and what its functional relevance is. 
The similarity between the profiles of lateral displacement of fibers and the force profiles of the whole muscle is striking. Similar to the force profiles, the curves of lateral translation also show a rather large displacement rate followed by a depression at short muscle lengths, and a slower rate at longer muscle lengths (Fig. 5D-F). Although this similarity has not been statistically evaluated, it suggests that displacement and deformation of the muscle and muscle force are more tightly related than muscle force and shortening of a single fiber. Consequently, although the sliding filament theory predicts a clear relation between fiber strain and force generation, the usefulness of fiber deformation as a measure for overall muscle behavior becomes doubtful. At the level of a complete muscle the relationship between deformation of a fiber and force generation is more complicated and becomes affected by numerous other factors, one of which is deformations originating from forces perpendicular to the direction of the considered fiber.

\section{CONCLUSION}

In this study, evidence has been generated that in muscle, forces perpendicular to the longitudinal direction of fibers on the muscle surface are present during contractions. Due to these forces muscles can rotate along their line of action and are not restricted to shortening along this line.

We started this study with the idea that muscle fibers are independent in the sense that they mainly transmit forces longitudinally and hardly interfere laterally with each other. Consequently, we hypothesized that fibers in an obviously inhomogeneous muscle like the $m$. plantaris will behave differently because they are of different lengths, but will still face similar global changes when the muscle contracts. Now we do not see any effect of morphologic asymmetry on the strains of different fibers. Rather, the lateral displacements of fibers in the muscle attract attention. It can be hypothesized that due to lateral interactions between fibers, differences in longitudinal strains are equilibrated, but that, consequently, lateral deformations have to arise.

\section{LITERATURE CITED}

Balnave CD, Allen DG. 1996. The effect of muscle length on intracellular calcium and force in single fibers from mouse skeletal muscle. J Physiol 492:3:705-713.
Benninghoff A, Rollhäuser H. 1952. Zur inneren Mechanik des gefiederten Muskels. Pflügers Archiv 254:527-548.

Callister RJ, Peterson EH. 1992. Design and control of the head retractor muscle in a turtle, Pseudemus (Trachemys) scripta: II. Efferent innervation. J Comp Neurol 325:422-434.

Eijden TMG J v, Raadsheer MC. 1992. Heterogeneity of fiber and sarcomere length in the human masseter muscle. Anat Rec 232:78-84.

Griffiths RL. 1991. Shortening of muscle fibers during stretch of the active cat medial gastrocnemius muscle: the role of tendon compliance. J Physiol (Lond) 436:219-236.

Huijing PA. 1996. Important experimental factors for skeletal muscle modelling: non-linear changes of muscle length force characteristics as a function of degree of activity. Eur J Morphol 34:47-54.

Huijing PA. 1999. Muscle as a collagen fiber reinforced composite: a review of force transmission in muscle and whole limb. J Biomech 32:329-345.

Huijing PA, Baan GC, Rebel GT. 1998. Non-myotendinous force transmission in rat extensor digitorum longus muscle. J Exp Biol 201:682-691.

Muijtens AMM, Roos JMA, Arts T, Hasman A. 1995. 3-D Reconstruction of marker position form stereo imagesracking markers with missing data by lower rank approximation. J Biomech 30:95-98.

Patel TJ, Lieber RL. 1997. Force transmission in skeletal muscle: from actomyosin to external tendons. In: Hollszy JO, editor. Exercise and Sport Sciences Reviews, vol 25. Baltimore: Williams and Wilkins. p 321-363.

Sacks RD, Roy RR. 1982. Architecture of the hind limb muscles of cats: Functional significance. J Morph 173:185-195.

Savelberg HHCM, Schamhardt HC. 1995. The influence of inhomogeneity in architecture on the modelled force-length relationship of muscles. J Biomech 28:187-197.

Scott SH, Loeb GE. 1995. Mechanical properties of aponeurosis and tendon of the cat soleus muscle during whole-muscle isometric contractions. J Morphol 224:73-86.

Street SF. 1983. Lateral transmission of tension in frog myofibres: a myofibrillar network and transverse skeletal cytoskeletal connections are possible transmitters. J Cell Physiol 114: 346-364.

Van Bavel H, Drost MR, Wielders JD, Huyghe JM, Huson A, Janssen JD. 1996. Strain distribution on rat medial gastrocnemius (MG) during passive stretch. J Biomech 29:1069-1074.

Van der Linden BJJJ. 1998. Mechanical modeling of skeletal muscle functioning. PhD. Thesis, The Netherlands: Twente University.

Van Donkelaar CC, Willems PJB, Muijtjens AMM, Drost MR. 1999. Skeletal muscle transverse strain during isometric contraction at different lengths. J Biomech 32:755-762.

Willems MET, Huijing PA. 1994. Heterogeneity of mean sarcomere length in different fibers: Effects on length range of active force production in rat muscle. Eur J Appl Physiol Occ Physiol 68:489-496

Woittiez RD, Huijing PA, Rozendal RH. 1983. Influence of muscle architecture on the length-force diagram. A model and its verification. Pflügers Archiv 397:73-74.

Zuurbier CJ, Huijing PA. 1993. Changes in geometry of actively shortening unipennate rat gastrocnemius muscle. J Morphol 218:167-180. 